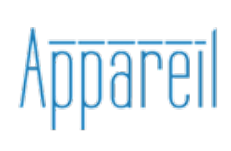

Appareil

$21 \mid 2019$

Le théâtre comme appareil

\title{
Théâtre, appareil(s), médias
}

Notes à propos de l'article de Sophie-Aurore Roussel, « Le théâtre, un appareil originaire?»

Jean-Louis Déotte $†$

\section{OpenEdition}

Journals

Édition électronique

URL : http://journals.openedition.org/appareil/3252

DOI : 10.4000/appareil.3252

ISSN : 2101-0714

Éditeur

MSH Paris Nord

Référence électronique

Jean-Louis Déotte t, « Théâtre, appareil(s), médias », Appareil [En ligne], 21 | 2019, mis en ligne le 11 juillet 2019, consulté le 30 juillet 2020. URL : http://journals.openedition.org/appareil/3252 ; DOI :

https://doi.org/10.4000/appareil.3252

Ce document a été généré automatiquement le 30 juillet 2020

\section{(c) $(1)(9)$}

Appareil est mis à disposition selon les termes de la Licence Creative Commons Attribution - Pas d'Utilisation Commerciale - Pas de Modification 4.0 International. 


\section{Théâtre, appareil(s), médias}

Notes à propos de l'article de Sophie-Aurore Roussel, « Le théâtre, un appareil originaire?»

Jean-Louis Déotte †

\section{Introduction}

1 Florence Dupont ${ }^{1}$ insiste sur un bouleversement général du rapport entre la culture théologico-politique athénienne et la mise en scène tragique. Si pour nous les textes des tragédies qui nous sont parvenus, via Alexandrie, sont quasi sacrés, leurs mises en scène, toujours renouvelées, sont de l'ordre d'une compétition ouverte entre metteurs en scène, qui ne laisse guère de traces. C'est le règne de l'impermanence qui caractérise la culture théâtrale, où l'oubli règne en maître, une mise en scène chassant l'autre. Le festival d'Avignon est ainsi devenu une foire aux mises en scène. Or, pour les Athéniens, contrairement à ce que l'on croit depuis le XIX ${ }^{e}$ siècle et les Romantiques, à la suite de la Poétique d'Aristote, ce n'était pas du côté des textes « tragiques » qu'il fallait chercher le code culturel de la Cité, sa philosophie et sa politique, car ces textes étaient destinés, une fois appris, à ne pas subsister (au contraire du code culturel athénien, du mythe et de l'épopée). C'est la raison pour laquelle ils étaient écrits. L'écriture, c'est l'oublieuse mémoire de Platon (le Phèdre). L'institution politico-philosophique était surtout l'affaire de la structure spatiale du théâtre, de son architecture, des masques, des rôles fixés des acteurs, de la musique... bref, du côté d'un rituel permanent dont tous les éléments constituent un appareil spécifique, analogique, dont la capacité consistait bien à produire des apparences esthétiques, par définition éphémères et événementielles : les pièces effectivement jouées, les textes, les chants, etc. C'est depuis la Poétique d'Aristote que nous sommes modernes (comme Jacques Rancière) et croyons que l'essentiel, le permanent, pour les Grecs, c'était les textes tragiques, d'où l'invention au XvIII ${ }^{\mathrm{e}}$ siècle d'un genre littéraire nouveau, le tragique. 


\section{L'appareil théâtral grec}

2 Sophie-Aurore Roussel décrit cet appareil théâtral grec qui, selon elle, constitue une sorte d'appareil originaire pour l'Occident ${ }^{2}$. Guy Debord avait raison de lier " société du spectacle » et machinerie théâtrale, même s'il avait tort de la décrire en termes péjoratifs (le spectacle serait le stade ultime du capitalisme). Les espaces y sont clairement hiérarchisés: le proskènion (proscenium) où évoluent les protagonistes (acteurs professionnels jouant les héros, là où se déroule le théâtre au sens classique), en haut de la skènè, le theologeion où s'expriment les dieux ouraniens grâce à la mèchanè, machine verticale qui permettait l'apparition des dieux en haut de la skènè. En bas de la skènè, l'ekkyklème, machine horizontale qui fait jaillir un plateau perpendiculaire au proskenion, vers le public, plate-forme roulante ou pivotante située dans l'axe de la porte principale de la skènè. Elle « route » à l'extérieur une partie de l'espace intérieur : elle peut montrer des scènes d'intérieur ou le résultat d'actions qui s'étaient déroulées à l'intérieur, ou faire apparaître des spectres, fantômes, divinités ouraniennes. Entre cette zone de jeu et le public, plus bas, se trouve l'orchestra où évolue le chœur, dans cette zone intercalaire justement. Au milieu de l'orchestra, la thymèle, autel de Dionysos.

Une machine théâtrale doit particulièrement retenir notre attention: l'ekkyklème. Si l'articulation de l'intérieur et de l'extérieur est indissociable d'une technique de présentation, n'est-ce point-là le rôle de cet appareil global ? Là où Walter Benjamin pouvait parler d'entrecroisement des espaces pour l'architecture moderne ou de compénétration, s'agissant de certains « matériaux » poreux comme la lave pour Naples ou le verre définitivement vierge de traces pour le Bauhaus, la mécanique était alors la clef des adresses.

4 L'autre machine, le theologeion, lieu d'expression des dieux ouraniens, est verticale et permet l'apparition des divinités en haut de la skènè. Articulation essentielle pour une pensée qui caractérise les Mortels à partir des Immortels, et non l'inverse. Nous avons donc deux machines théâtrales : comment la perspective faisant renaître le théâtre antique avec le théâtre Olympique (teatro Olimpico) à Vicences ${ }^{3}$, va-t-elle les interpréter?

\section{Fondation d'une famille d'appareils}

5 Si le théâtre antique est l'appareil originaire de l'Occident, ce qui exclut les autres scènes, non occidentales, mais aussi les Mystères médiévaux, alors on devrait en retrouver les caractéristiques essentielles, les espaces hiérarchisés et les machines, parmi tous les appareils «secondaires", comme les appareils projectifs surgis à la Renaissance.

6 Ce que le théâtre antique et les appareils projectifs ont en commun, c'est leur commune appartenance à une cosmétique, à l'ordre symbolique «délibératif ${ }^{4}$ ». Ce qui vient immédiatement à l'esprit du côté de la Grèce, c'est la structure dialogique des textes de Platon. Du côté de la Renaissance italienne, qui voit surgir la perspective comme un appareil tout armé, on pense à la démocratie (Machiavel) comme ce «régime » qui légitime le combat politique. Peut-être qu'après avoir fait place aux différentes inversions, sur le modèle du carnaval, l'analogie entre scène théâtrale et public devrait s'ouvrir aux figures de la stasis, la guerre civile, si bien analysées par Nicole Loraux? 
7 Si le chœur antique a cette fonction mémorielle de réunification de la société politique, alors son analogue renaissant, c'est la représentation en peinture, l'istoria peinte, appareillée par la perspective ${ }^{5}$.

\section{Le différend Visible/Lisible : Aristote via Averroès via Borges}

8 Il y a une bonne méthode pour caractériser cette cosmétique travaillée par le hiatus entre scène et public, c'est de la confronter à une autre cosmétique, celle de la révélation. Sa spécificité est bien cernée par Jorge Luis Borges ${ }^{6}$ dans un des contes de L'Aleph. La Quête d'Averroès?.

[...] Aristote. Ce Grec, source de toute philosophie, avait été accordé aux hommes pour leur enseigner tout ce qui se peut savoir ; interpréter ses ouvrages comme font les ulémas le Coran, était la difficile entreprise que se proposait Averroès. [...] aux difficultés intrinsèques s'ajoutait le fait qu'Averroès, ignorant du syriaque et $d u$ grec, travaillait sur la traduction d'une traduction. La veille deux mots douteux l'avaient arrêté au seuil de la Poétique. Ces mots étaient tragodia et comœdia ${ }^{8}$.

Puis l'un des convives de la soirée à laquelle participe Averroès, un voyageur nommé Aboulkassim, narra ce qui suit :

_ Les actes des fous, dit Farach, dépassent les prévisions du sage.

_ Ils n'étaient pas fous dut préciser Aboulkassim. Ils étaient en train, me dit un marchand, de représenter une histoire. [...]

_ Ces personnes parlaient-elles? demanda Farach.

_ Bien sûr, elles parlaient, dit Aboulkassim [...]

_ Dans ce cas, conclut Farach, il n'était pas besoin de vingt personnes. Un seul

narrateur peut raconter n'importe quoi, quelle qu'en soit la complexité 9

En quelques lignes, la spécificité de l'Occident pour les hommes du Livre est pointée. Car comme le conteur est étranger au monde du théâtre, et donc à la figure qui traverse et constitue le visible, il ne peut que décrire sans le comprendre ce que nous appelons jeu (agon) entre des protagonistes sur une scène. Pour lui et ses compagnons, dont Averroès, le conte, c'est-à-dire le langage, suffit à tout.

Mais pour l'Occidental, il en va tout autrement, car pour le dire en termes lyotardiens ${ }^{10}$, entre la figure (le figural) et le langage articulé, il y a un différend infranchissable. C'est le différend entre le visible d'une part, l'intelligible d'autre part. Pour Jean-François Lyotard, et contrairement à Jacques Rancière ${ }^{11}$ qui est strictement aristotélicien, le langage se trouve face à une altérité, celle du visible. C'est ce qui légitime pour nous le théâtre, les actions, les postures, les gestes, les cris et les silences, etc. Il y a un fossé ${ }^{12}$ infranchissable entre l'épaisseur du visible que décrit Merleau-Ponty (la différence focale) et le système horizontal des signes qui structure le langage articulé. La figure (visible ou lisible) est irréductible au système de signes. L'Islam aurait radicalement ignoré la figure (visible) parce qu'il l'aurait transférée dans la poésie et dans l'épaisseur du Texte sacré. Pour l'Islam, la figure ne peut être que d'ordre textuel, comme en Occident, la rhétorique et la poétique, la césure du vers chez Hölderlin par exemple. Les fenêtres de ses édifices ne sont pas des plans de projection, mais des pare-soleil finement ouvragés (moucharrabieh), ses édifices sont recouverts de structures géométriques dont les éléments sont composables selon quelques principes de base (muquarnas) ${ }^{13}$. Il y a bien une théorie de la vision (rectiligne) qui précède de loin la science occidentale, mais c'est, là aussi, au nom de la rationalité mathématique ${ }^{14}$. Bref, 
un déni de la figure par la mathématisation du visible : preuve de l'essence divine du monde. Entre cette cosmétique et l'écriture numérique, il n'y a qu'un pas, sans avoir à franchir l'ère de l'écriture projective.

11 Il y a même, selon Borges, dans le cas d'Averroès, du haut d'un régime symbolique qui ignore radicalement le figural (visible), un tort qui est infligé dans sa tentative de traduction d'Aristote :

Quelque chose lui avait révélé le sens des deux mots obscurs. D'une ferme et soigneuse calligraphie, il ajouta ces lignes à son manuscrit: "Aristû (Aristote) appelle tragédie les panégyriques et comédie les satires et anathèmes. D'admirables tragédies et comédies abondent dans les pages du Coran et dans les moallakas du sanctuaire ${ }^{15}$.

Il y a tort, nous explique Le Différend de Lyotard, quand un ordre symbolique s'impose à un autre en ne reconnaissant pas sa légitimité. Bien sûr, on pourrait imaginer la place d'un juge interculturel en position de surplomb, capable de restituer à chaque ordre symbolique sa légitimité, mais cette place n'existe pas car où trouverait-elle sa propre légitimité?

Ainsi Aristote sera victime d'un tort, car Borges ne peut pas occuper ce point de surplomb grâce à quoi le coup de force d'Averroès serait révélé. Car, pour cela, il faudrait qu'il puisse sortir du régime du conte :

[...] à mesure que j'avançai [...] je compris que mon œuvre se moquait de moi. Je compris qu'Averroès s'efforçant de s'imaginer ce qu'est un drame, sans soupçonner ce qu'est un théâtre, n'était pas plus absurde que moi, m'efforçant d'imaginer Averroès, sans autre document que quelques miettes de Renan, de Lane, d'Asin Palacios. Je compris, à la dernière page, que mon récit était un symbole de l'homme que je fus pendant que je l'écrivais et que, pour rédiger ce conte, je devais devenir cet homme et que, pour devenir cet homme, je devais écrire ce conte, et ainsi de suite à l'infini ${ }^{16}$.

Est-ce que Borges aurait réussi si son dossier « Averroès » avait été mieux fourni ? C'est l'illusion de Fustel de Coulanges écrivant la Cité antique (1864) : l'illusion que l'historien peut faire revivre l'intégralité de ce qui a existé "comme si vous y étiez ». L'illusion d'un possible savoir absolu, celle de la passion réalisée de l'archiviste. On ne peut saisir dans sa vérité que ce qui a été appareillé de même. Nous pouvons imaginer ce qu'était le théâtre antique non seulement parce que nous en connaissons les ruines, les textes, les auteurs, le contexte socio-historique, les philosophes, etc., mais essentiellement parce que nous avons une expérience actuelle du jeu théâtral. Il en irait de même pour une peinture en perspective qui a désormais besoin du musée universel. C'est ce sol commun qui rend possible la traduction. Sans expérience appareillée de même, il n'y a point de sol commun. Borges prend acte de son échec : pas plus qu'Averroès ne pouvait comprendre Aristote, pas plus il ne pourra accomplir la destination d'un musulman.

\section{L'appareil originaire et ses appareils secondaires}

L'appareil originaire permettrait d'expliquer la survie de la scène théâtrale et son retour, que ce soit dans la peinture perspectiviste, la photographie, le cinéma, l'exposition, etc., sans compter tous les usages possibles de la mise en scène. S'il y a toujours du théâtre filmé, par exemple chez Alain Resnais (Smocking, No smocking, etc), c'est certes que le cinéma est cet appareil qui absorbe tous les autres, mais qu'on ne se débarrasse pas si facilement de la matrice de l'Occident. La distinction «appareil 
originaire »/«appareil secondaire » (ou «appareil proprement dit» ou "appareil après-coup ») ne cache pas sa filiation freudienne. C'est Freud qui à propos du refoulement, comme de la scène ou du fantasme (originaires), a introduit la distinction entre l'« originaire » ou «primitif» et le « secondaire » ou «à proprement dit ». Il n'y aurait pas de refoulement secondaire s'il n'y avait eu déjà des éléments refoulés, originaires, lesquels «attirent " vers le fond les éléments récents que le conscient ne saurait admettre.

Par conséquent, le théâtre ne s'inscrit pas seulement dans une généalogie, dans une succession : dans la famille des appareils projectifs, il ne serait donc pas seulement le premier, mais le fondement de tous les autres appareils projectifs. Et ce, parce qu'il serait le premier appareil à faire droit à la figure, sous la forme de la rampe, sous la forme de l'articulation entre l'adresse horizontale et la verticale, sous la forme des machines mises en œuvre pour ce faire, sous la forme de la distinction face/profil, sous la forme de l'inversion des rôles, sous la forme de la représentation qui rend possible et confirme la légitimité de la présence, etc.

Peut-on penser qu'il fonde la famille projective et que les appareils secondaires en sont les déterminations époquales? Configuré à nouveaux frais par la perspective par exemple, ou par le cinéma, déterminé et déterminant, chaque époque appareillée le transforme tout en le conservant dans ses principes constituants.

La thèse serait la suivante: tous les appareils projectifs, depuis la camera obscura, tiennent du théâtre antique parce qu'ils sont, d'une manière ou d'une autre, analogiques ${ }^{17}$. Mais ils n'entretiennent pas cet héritage de la même manière: la perspective est projective parce que sur l'écran ou support d'inscription (le «tableau » selon Alberti), les traces de la nature perdue viennent s'inscrire par l'intermédiaire de la main, sans imiter la nature (Dioptrique de Descartes). Avec l'appareil photographique, l'inscription projective est optico-chimique (Niepce) et reproductible (Talbot). Avec le musée, la projection est rétroactive, imaginative (l'œuvre récente inventant son archive pour un public "averti»). Avec la psychanalyse, la projection est psychologique, inconsciente et réversible. Avec la vidéo, elle est électronique, consciente et irréversible. Si le cinéma est, comme l'écrit Benjamin, "Démêlement (résultat?) de toutes les formes de vision, de tous les rythmes et de tous les temps préformés dans les machines actuelles, de telle sorte que tous les problèmes de l'art actuel ne peuvent trouver leur formulation définitive qu'en corrélation avec le film ${ }^{18}$ ", alors il va conjuguer les modes de la projection, et donc de l'analogie, de bien des manières.

19 On pourrait suggérer qu'en ses débuts, un appareil projectif est comme brut de décoffrage: ses constituants propres sont explicites. Ce n'est qu'après avoir exploré toutes ses possibilités créatrices qu'il laisse l'appareil originaire revenir. Il y a une théâtralisation progressive des appareils projectifs. C'est le chemin qui mène de Masaccio à Ingres, de Niepce à Doisneau, des frères Lumière à Truffaut.

Conclusion: cet appareil originaire introduirait un nouvel ordre symbolique, une nouvelle cosmétique, un nouveau genre de discours (le délibératif), une nouvelle surface d'inscription permanente (l'ensemble architectural décrit plus haut). 


\section{L'appareil originaire et Platon}

21 On se demandait antérieurement ce qu'avait été l'appareil dominant des Grecs, on pensait avoir une piste avec la notion de skiagraphia (la projection d'ombres sur une paroi) et on était conforté par le mythe de Débutade comme origine du dessin, ou plutôt de la silhouette. Or, il est probable que cette technique projective était utilisée pour les décors de théatre. Si on a donné tant d'importance au «mythe » de la Caverne chez Platon (La République), c'est qu'on a été obnubilé par ce «théâtre d'ombres » alors qu'on faisait l'impasse sur sa condition de possibilité même : le théâtre athénien. On a pris l'élément pour le tout, un élément du décor pour la machine globale. La philosophie de Platon se développe sur l'oubli de cet appareil originaire. Peut-être que la khôra est un souvenir de cette fondation oubliée?

Or notre appareil originaire est autrement plus complexe (plus différencié, plus articulé, etc.) que la seule khôra du Timée (ce support de tous les lieux possibles, ce porte-empreintes, ce réceptacle des Idées les portant à l'empirie, cet engin de sélection, etc.).

\section{L'appareil originaire et la temporalité}

L'appareil originaire introduit un rapport décisif à la temporalité : non pas la répétition du Même, le destin, l'éternel retour, mais son interruption, grâce à laquelle quelque chose comme une émancipation du temps est concevable.

Dans sa correspondance avec Florens Christian Rang, Walter Benjamin revient sur les rapports entre agon, carnaval et tragédie et demande à celui-ci si d'autres preuves « scientifiques » vont dans le sens d'une « sortie » du théâtre hors du cercle astral. À ses yeux, la seule racine agon dans « protagoniste » n'est pas suffisante. Rang lui répond le 28 janvier 1924 (Théâtre et Agon).

Je n'ai aucune documentation pour prouver que la tragédie dérive de l'agon. Mais outre le mot "(Prot)agoniste", j'attire ton attention sur le char de Thespis, le «carnaval» qui suit la révolution céleste des astres, non pas l'ordre fixé (en astrologie), mais quand il éclate (dans le temps intercalaire), de sorte que l'extase peut jaillir de l'angoisse ; la parole libre (dictamen) peut se passer de la loi : le dieu nouveau (Dionysos) peut réduire les anciens. [...] La tragédie est rupture de l'astrologie [en ital. chez Rang] et donc l'affranchissement du sort fixé par les astres ${ }^{19}$.

Puis Rang met en relation le procès judiciaire et le théâtre : «le dialogue est une joute oratoire, c'est-à-dire, compétition ${ }^{20}$. Si une caractéristique essentielle du théâtre, c'est d'être cet appareil originaire qui brise le destin astrologique, alors il ne faut pas chercher ailleurs la puissance émancipatrice. Dès lors, les appareils proprement dits, en particulier les projectifs, seront écartelés entre la puissance destinale, une cosmétique, et une émancipation esthétique.

\section{Les appareils ne sont pas des médias de communication}

C'est ce qui fait toute la différence entre la philosophie des appareils et la théorie de la communication, laquelle avec Friedrich Kittler par exemple ${ }^{21}$, ignore l'esthétique, c'est- 
à-dire l'émancipation. L'appareil originaire, réduit à sa structure, prépare ce qui dans la modernité projective sera strictement de l'ordre de la communication, laquelle selon Friedrich Kittler est indissociable de la guerre, comme chez Paul Virilio. Kittler ne s'intéressera pas à l'immense production esthétique rendue possible par la perspective, mais au progrès dans la visée de l'artillerie ou aux utopies géométriques de villes fortifiées ${ }^{22}$.

Un médium de communication, c'est un appareil qu'on a séparé de son esthétique. En tant que tel, il n'émancipe de rien, mais il enferme la singularité et l'être en commun dans des rôles prédéterminés, dont la théorie fut établie par Shannon. Un auditeur ou un téléspectateur n'a d'autres choix que celui de choisir son programme. Les contenus artistiques générés par les médias de communication sont rares, de l'ordre du détournement.

En tant que médium, il fixe ontologiquement le statut du destinataire. On peut réduire l'appareil photo à sa capacité policière d'établir des identités, c'est le côté sous-appareil d'État que retient l'althussérien Vilém Flusser ${ }^{23}$. En tant que message (ou contenu), la photo est déterminée par le médium. En fait, la relation message/medium est une manière nouvelle d'articuler matière/forme. Le medium réinvestissant les diverses formes de finalité de la forme.

On peut aussi insister sur la nouvelle temporalité qui surgit avec lui. Or, l'esthétique est indissociable de ces formes de temporalité non répétitives.

\section{Analogie et projection}

L'article de Sophie-Aurore Roussel met l'accent sur le théatre athénien comme appareil et renvoie aux ouvrages de Florence Dupont. La place de l'analogie y est centrale, non l'analogie au sens des Médiévaux interprétant Aristote à partir d'Avicenne et/ou Averroès (la participation des qualités à la substance), mais l'analogie au sens moderne. Pour la science, il y a analogie quand il y a identité quantitative de rapports. Par exemple quand on peut mettre en relation un grand rectangle et un petit. Il y a une forme géométrique commune, même si les dimensions changent. Il y a analogie quand on peut écrire $: a / b=c / d=e / f$, etc. Plus précisément :

Selon Kant, l'analogie signifie "non pas, comme on l'entend communément, une ressemblance imparfaite entre deux choses, mais une ressemblance parfaite de deux rapports entre des choses tout à fait dissemblables ». Au moyen de l'analogie, je peux "donner un concept relatif de choses qui me sont absolument inconnues ». Ainsi puisje penser «le rapport des choses en soi au monde sensible de façon analogique au rapport entre les choses de ce monde (les phénomènes) $»^{24}$. L'analogie est donc une procédure de description de l'inconnu (la quatrième proportionnelle), sans pouvoir en déduire l'existence de cet inconnu. Pour Kant, l'existence se constate toujours au moyen des intuitions sensibles.

31 "Tous les phénomènes sont soumis a priori, quant à leur existence, à des règles qui déterminent leurs rapports entre eux dans un temps ». Alors que dans le domaine des mathématiques, les analogies «sont des formules qui expriment l'égalité de deux rapports quantitatifs ", l'analogie dans la philosophie signifie l'égalité de deux rapports qualitatifs « dans lesquels, à partir de trois membres, je ne puis connaître et donner a priori que le rapport à un quatrième, mais non ce quatrième membre lui-même ; j'ai 
bien cependant une règle pour le chercher dans l'expérience, et une marque pour l'y découvrir ". Les analogies de l'expérience valent de "manière non constitutive, mais simplement régulatrice", elles sont des principes de l'usage «empirique » et non «transcendantal $»^{25}$ de l'entendement. Elles ne déterminent pas les phénomènes et la synthèse de leur intuition empirique, mais seulement l'existence et leur rapport entre eux, relativement à cette existence.

\section{Analogies internes et externes aux tragédies} partir du moment où elle peut isoler, dans chaque pièce, des rapports comparables. Un rapport est structurel, comme celui qui oppose les héros qui sont habités par l'hubris (le déséquilibre, la tension, la crise, etc.) et ceux qui ont atteint un état de sagesse, de connaissance, d'équilibre. C'est la vieille opposition Dionysos/Apollon qui serait déterminante. Ou encore celle du cortège carnavalesque avec le retour à l'ordre. Le désordre du carnaval redonne toute l'énergie nécessaire à un nouvel ordre ${ }^{26}$, ou encore, il permet l'initiation d'une classe d'âge ${ }^{27}$.

Parallèlement, ou analogiquement, les spectateurs ne peuvent que se projeter dans ces processus mis en scène, au-delà de la rampe. Comme la tragédie ne se réduit pas à un contenu de texte, la fabula, contrairement au coup de force imposé par Aristote, c'est sur le contexte historique et sur la réception que porte l'essentiel. La première série de rapports «tragiques » a pour analogue la seconde série des conversions de la réception par le public. Mais le mouvement de conversion a été lancé par les acteurs sur scène : c'est ce qui permet à Sophie Roussel d'éviter le terme de catharsis. La catharsis n'étant à l'œuvre que dans des tragédies générées par la Poétique d'Aristote qu'on peut dès lors considérer comme un appareil au second degré. Un appareil théorique qui vient reconfigurer la première série des pièces tragiques, qu'on pourrait appeler «originaires» (Euripide, Sophocle, etc.). Suivant cette pente, les pièces postaristotéliciennes seraient "secondaires ", «à proprement parler «tragiques »", servant alors de modèles à l'art théâtral classique. reprendre la fiction freudienne d'un premier $\mathrm{Moïs}^{29}$ imposant à un peuple rétif les Tables de la Loi, succombant à la suite d'une révolte païenne (le culte du Veau d'or), mais ressurgissant sous la forme fantomale, dite historique, d'un Moïse devenu respectable et fondateur de religion. Il y aurait eu un Moïse "originaire», puis un Moïse « à proprement parler ».

Si la seconde série, celle des spectateurs, est mise en branle par la première, c'est que dans un premier temps, il y a eu projection (identification) du peuple athénien sur l'appareil théâtral, puis réappropriation après la réification des processus joués par son double.

On voit que les catégories utilisées par Edgar Morin pour analyser les rapports de l'homme à son double imaginaire ${ }^{30}$ à partir du cinéma, ont leur légitimité pour analyser le théâtre pré-aristotélicien. Dès lors, même si ce théâtre peut relever d'un appareil originaire, ce n'est qu'à partir du plus récent des appareils secondaires (le cinéma) qu'on peut l'analyser, en vérité. Il y a donc un cercle entre l'originaire et le secondaire.

Appareil, 21 | 2019 
L'originaire est la condition de possibilité du secondaire qui, en retour, le rend intelligible.

\section{Quand le théâtre antique devient humaniste}

Si l'analogie est au cœur de la projection, c'est, pour la perspective, sous la forme de la proportionnalité des triangles ${ }^{31}$. C'est dire que la projection à l'œuvre sur une scène athénienne, était globale (c'est tout un public qui se projetait sur ses héros), alors que quand l'appareil secondaire perspectif impose sa détermination à la scène comme à Vicenza, c'est une projection quantitative (quantifiable) et non plus qualitative qui s'impose. La rampe, véritable plan de symétrie, fait le partage entre les gradins du public et l'orchestra, la skènè est construite selon les lois de la perspective, des rues sont percées dans le décor urbain où les acteurs peuvent se mouvoir (cf. schémas et maquettes de Scamozzi).

Qu'est-il arrivé à l'Edipe de Sophocle, première pièce jouée à Vicenza et donc historiquement, première pièce de l'Antiquité rejouée sur une scène en perspective ? Cela revient à aller au cœur de ce qu'on appelle le premier Humanisme : reprendre des textes de l'Antiquité en les mettant en perspective. Ce qui a au moins deux significations: cette adaptation doit respecter la différence des temps comme le fit Machiavel avec ses Discours sur la première Décade de Tite Live, Florence n'est pas Rome, la démocratie moderne n'est pas la république, mais Florence démocrate peut se comprendre dans la confrontation avec Rome républicaine. La réflexivité a pour condition l'altérité. Les luttes entre le peuple et les grands, dans les deux cas sont intelligibles, les uns ne voulant pas se laisser dominer, les autres voulant dominer. Donc, du point de vue de la domination, il y a une analogie entre Rome et Florence, à condition de respecter leurs différences. D'autant que la conséquence est la même : la lutte politique est le terreau d'où surgissent les lois qui affirment la liberté selon Machiavel.

Seconde signification : la nouvelle mise en scène (ce qui englobe tous les éléments du "théâtre ») doit respecter la consistance de l'écriture projective que décrit fort bien Descartes. On peut postuler que là où il y avait image (au sens le plus large, ce qui va jusqu'à la métaphorisation) s'impose une écriture qui ne ressemble à rien. Ce qui devrait avoir des conséquences considérables pour la fabula, ce qui est visible dans l'ordre pictural. Que reste-t-il du contenu chrétien après sa mise au carreau? Le percement de la skènè n'est que la partie visible de l'iceberg !

\section{Nature de l'écriture projective : Descartes}

\footnotetext{
Il faut au moins que nous remarquions qu'il n'y a aucune image qui doive en tout ressembler aux objets qu'elles représentent : car autrement, il n'y aurait point de distinction entre l'objet et son image : mais qu'il suffit qu'elles leurs ressemblent en peu de choses; et souvent même que leur perfection dépend de ce qu'elles ne leur ressemblent pas tant qu'elles pourraient faire [...].

Or, il faut que nous pensions tout de même des images qui se forment dans notre cerveau, et que nous remarquions qu'il est seulement question de savoir comment elles peuvent donner moyen à l'âme de sentir toutes les diverses qualités des objets auxquels elles se rapportent, et non point comment elles ont en soi leur ressemblance ${ }^{32}$.
} 
Par conséquent, l'écriture projective tend à réduire la figure visible à un état langagier, elle est grosse de l'écriture numérique. Ce qui est un véritable défi pour le théâtre actuel, comme pour les "arts vivants ». La rampe comme plan de symétrie rendait réversibles le point de vue du public et le point de fuite. Architecturalement face au demi-cercle du «théâtre » se trouve le rectangle de la scène. La fabula était soumise au point de fuite qui était aussi le point du sujet; c'est dire qu'elle était habitée par le sujet, qu'elle était subjectivée. À partir de ce moment, les pièces furent écrites de ce point de vue. Ce qui n'est plus le cas avec les «écrivains de plateau » du théâtre contemporain.

41 La dimension verticale qui était mise en œuvre par une machine fut réduite à la proportionnalité horizontale, les dieux ne furent plus des immortels, mais des représentations. Seules quelques statues monumentales, intégrées dans la façade du décor, rappelèrent qu'il y avait un temple et une dimension de transcendance. Que reste-t-il des dieux quand le principe de la rampe a été relégué ? La stricte immanence d'une chambre d'échos?

42 La conception projective des rapports publics/mise en scène globale, permettait de résoudre une des grandes difficultés de la pensée politique classique. Que ce soit chez Hannah Arendt (Qu'est-ce que la politique ?) ou chez Jean-Luc Nancy (Être, singulier,

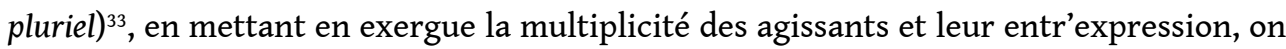
sortait certes d'une problématique de l'intersubjectivité avec toutes ses apories (comment sortir du sujet pour reconnaître une autre subjectivité ?), mais on se privait d'une articulation fondamentale. Car passer d'Un au Deux, ce n'est pas un grand progrès si l'on ne s'interroge pas sur ce qu'il y a entre eux: le milieu des appareils. Comment expliquer qu'il y a de l'histoire si l'on en reste au face-à-face entre une singularité et une autre? Le théâtre athénien était bien le "double " grâce à quoi la société politique s'était constituée. Il en ira de même avec la multiplicité des représentations où la société florentine s'est configurée, probablement à partir de la révolte des Ciompi (xIv ${ }^{\mathrm{e}}$ siècle) et de la constitution du « cercle de Brunelleschi ${ }^{34}$ » au $\mathrm{XV}^{\mathrm{e}}$ siècle.

Mais un pouvoir politique qui arriverait à saturer par sa fantasmagorie propre le milieu des appareils et des médias de communication se condamnerait nécessairement à la désintégration entropique : n'est-ce point le destin d'un monde esthético-politique où la figure ayant disparu, le langage se trouverait face à du langage ?

\section{BIBLIOGRAPHIE}

Belting Hans, Florence et Bagdad. Une histoire du regard entre orient et occident, traduit de l'allemand par Naïma Ghermani et Audrey Rieber, Paris, Gallimard, 2012.

Benjamin, Walter : Paris, capitale du XIX $x^{e}$ siècle, Paris, éditions du Cerf, 1989, trad. fr. Lacoste.

Clastres Pierre, La société contre l'État, Paris, Éditions de Minuit, 1974. 
Borges Jorge Luis, La quête d'Averroès, in L'Aleph. La quête d'Averroès, Paris, Gallimard, 1967, trad. fr. Caillois.

Daney Serge, La Rampe, Paris, Le Seuil, 1970.

Déotte Jean-Louis, L'époque de l'appareil perspectif. Brunelleschi, Descartes, Machiavel, Paris, éditions L'Harmattan, 2001.

Déotte Jean-Louis, L'Époque des appareils, Paris, éditions Lignes/Léo Scheer, 2004.

Déotte Jean-Louis, Qu'est-ce qu'un appareil ?, Paris, éditions L'Harmattan, 2007.

Déotte Jean-Louis, « Lyotard, penseur du différend culturel », Appareil [En ligne], 10 | 2012, mis en ligne le 09 février 2008, consulté le 25 juillet 2019. URL : http://journals.openedition.org/ appareil/115 ; DOI : 10.4000/appareil.115

Déotte Jean-Louis, « Quand Kittler renouvelle la métaphysique », Appareil [En ligne], 19 | 2017, mis en ligne le 21 décembre 2017, consulté le 26 juillet 2019. URL : http:// journals.openedition.org/appareil/2543; DOI : 10.4000/appareil.2543

Descartes René, Dioptrique [1637], in CEuvres complètes, t.4, Paris, Gallimard, coll. « Tel », 2009.

Dupont Florence, L'Insignifiance tragique, Paris, Le promeneur, 2001.

Dupont Florence, Aristote ou le vampire du théâtre occidental, Paris, Aubier, 2007.

Flusser Vilém, Philosophie de la photographie, Oberhausbergen, éditions Circé, 1996.

Freud Sigmund, trad. de l'allemand par Anne Berman, Paris, Gallimard, 1967.

Kant Emmanuel, Prolégomènes, § 58 et note, AK IV, 357, 221. Académie de Berlin.

Kittler Friedrich, Médias optiques. Cours berlinois, 1999, Paris, éditions L'Harmattan, trad. sous la dir. d'Audrey Rieber, 2015.

Lyotard Jean-François, Discours, Figure, Paris, éditions Klincksiek, 1971.

Marin Louis, Le Portrait du roi, Paris, Éditions de Minuit, 1981.

Morin Edgar, Le cinéma ou l'homme imaginaire. Essai d'anthropologie, Paris, Éditions de Minuit, 1956.

Rang Florens.Christian, Psychologie historique du carnaval [1927], Toulouse, éditions Ombres, 1990, trad. fr. Rey.

Roussel Sophie-Aurore, « Le théâtre, un "appareil originaire” ? , Appareil [En ligne], Articles, mis en ligne le 24 octobre 2016, consulté le 24 juillet 2019. URL : http://journals.openedition.org/ appareil/2351 ; DOI : 10.4000/appareil.2351 Wikipédia : note sur Le Palladio et le théâtre Olympique de Vicenza.

Sophie-Aurore Roussel, « Le théâtre, entre appareil et dispositif », Appareil [En ligne], 21 | 2019, mis en ligne le 29 juillet 2019, consulté le 29 juillet 2019. URL : http://journals.openedition.org/ appareil/3149

\section{NOTES}

1. Florence Dupont, L'Insignifiance tragique, Paris, Le promeneur, 2001.

2. Voir Sophie-Aurore Roussel, «Le théâtre, un « appareil originaire »?», Appareil [En ligne], Articles, mis en ligne le 24 octobre 2016, consulté le 24 juillet 2019. URL: http:// journals.openedition.org/appareil/2351; DOI : 10.4000/appareil.2351 
3. À propos du théâtre Olympique, voir l'article «Teatro Olimpico » sur Wikipedia (consulté le 11 juillet 2019). On y apprend notamment que: «La construction commença en 1580, l'année même où mourut Palladio, mais les travaux continuèrent sur la base de ses notes, sous la direction de son fils Silla. Ils furent achevés en 1584, comprenant la cavea, la loggia et le proscenium. Le problème restait donc posé de la réalisation de la scène en perspective, dont le principe avait été retenu par l'académie, mais pour laquelle Palladio n'avait laissé aucun véritable projet. Il fut donc fait appel à l'architecte Vincenzo Scamozzi, disciple de Palladio. Scamozzi dessina les décors de bois, dont les effets de perspective et le soin du détail firent grande impression lors du spectacle inaugural, apportant en outre quelques adaptations et achèvements nécessaires au projet de Palladio. Les salles contiguës de l'Odèo et l'Antiodèo, ajoutées au projet original du portail d'entrée, sont attribuées à Scamozzi. »

4. Paradoxalement, c'est la seule cosmétique " anarchique ", dans la mesure où elle a la capacité de remettre en cause son propre fondement cosmique (le Big Bang) et ses propres frontières.

5. Je renvoie ici à mon ouvrage L'Époque de l'appareil perspectif (2001) où je compare la peinture de représentation à un masque unifiant, une sorte de moi-idéal pour la cité florentine. La représentation devant être entendue comme ce qui présente et légitime la présence. Sur ce point, l'interprétation XVII ${ }^{e}$ siècle français par Louis Marin est indispensable (Le Portrait du roi, 1981). Car la métaphore du miroir, comme celle de « l'échange à l'infini du sujet et de l'objet » que Foucault croit pouvoir déduire de son analyse des Ménines (Les Mots et les Choses), sont bien trompeuses, car pour qu'il y ait un fidèle reflet, encore faut-il que l'objet soit constitué, c'est-à-dire qu'il ait une consistance physique, ce qui n'est précisément pas le cas d'une société politique. Si la production théâtrale athénienne comme la production plastique florentine furent si abondantes, c'est bien parce qu'il n'y a pas de « corps » politique (le « corps » étant aussi une mauvaise dérivation de la cosmétique de l'incarnation chrétienne). Bref, l'appareil ne génère pas des reflets d'un collectif objectivé, mais il contribue à l'institution configurée d'un être-ensemble qu'aucune science ne peut saisir.

6. Nous remercions Gerald Wildgruber pour les retrouvailles avec ce texte !

7. Jorge Luis Borges, L'Aleph. La quête d'Averroès, Paris, Gallimard, 1967, trad. fr. Caillois.

8. Ibid., p. 118-119.

9. Ibid., p. 123-125.

10. Jean-François Lyotard, Discours, Figure, Paris, Klincksiek, 1971.

11. Nous renvoyons ici à notre ouvrage Qu'est-ce qu'un appareil ?, Paris, L'Harmattan, 2007, ainsi qu'à notre article « Lyotard, penseur du différend culturel », Appareil [En ligne], 10 | 2012, mis en ligne le 09 février 2008, consulté le 25 juillet 2019. URL: http://journals.openedition.org/ appareil/115 ; DOI : 10.4000/appareil.115

12. Au théâtre, comme au cinéma, ce fossé peut être la fosse d'orchestre ou tout simplement la rampe, voir Serge Daney, La Rampe, Paris, Le Seuil, 1970.

13. On notera que deux édifices de Jean Nouvel à Paris rendent hommage à cette cosmétique : l'Institut du Monde arabe et la Philarmonie. Sans oublier les projets de Musée du Louvre d'Abu Dhabi, du Musée du Qatar, du Musée de l'Islam à New York.

14. Hans Belting, Florence et Bagdad. Une histoire du regard entre orient et occident, traduit de l'allemand par Naïma Ghermani et Audrey Rieber, Paris, Gallimard, 2012.

15. Jorge Luis Borges, L'Aleph, La quête d'Averroès, op. cit., p. 128.

16. Ibid., p. 129-130.

17. La notion d'« appareil » ne se limite pas à la capacité analogique de mettre en rapport des rapports. Chaque cosmétique, chaque régime du symbolique, doit appareiller la surface d'inscription des signes : la cosmétique « sauvage » de la narration doit appareiller le corps des hommes lors des rituels d'initiation des adolescents (voir Pierre Clastres, La société contre l'État, Paris, Éditions de Minuit, 1974), la cosmétique de la révélation peut avoir comme appareil privilégié la cathédrale (christianisme) ou le Livre (Islam), etc. 
18. Walter Benjamin, Paris, capitale du XIXe siècle, Paris, éditions du Cerf, p. 412.

19. Florens Christian Rang, Psychologie historique du carnaval, 1927, Toulouse, éditions Ombres, 1990, trad. fr Rey.

20. Ibid., p. 305.

21. Friedrich Kittler, Médias optiques. Cours berlinois, 1999, Paris, L'Harmattan, trad. sous la dir. d'Audrey Rieber, 2015.

22. Jean-Louis Déotte, « Quand Kittler renouvelle la métaphysique », Appareil [En ligne], 19 | 2017, mis en ligne le 21 décembre 2017, consulté le 25 juillet 2019. URL: http:// journals.openedition.org/appareil/2543; DOI : 10.4000/appareil.2543

23. Vilém Flusser, Philosophie de la photographie, Oberhausbergen, éditions Circé, 1996.

24. Kant Emmanuel, Prolégomènes à toute métaphysique future, $\$ 58$ et note, AK IV, 357, p. 221. Académie de Berlin.

25. Ibid., 915-918, p. 159-161.

26. Alenjandro Jodorowsky, La Poésie sans fin, 2016.

27. Van Gennep, Les Rites de passage, 1909.

28. Florence Dupont, Aristote ou le vampire du théâtre occidental, Paris, Aubier, 2007.

29. Sigmund Freud, Moïse et le monothéisme, trad. de l'allemand par Anne Berman, Paris, Gallimard, 1967.

30. Edgar Morin, Le cinéma ou l'homme imaginaire. Essai d'anthropologie, Paris, Éditions de Minuit, 1956.

31. Abraham Bosse, Manière universelle de Mr Desargues, pour pratiquer la perspective par petit-pied, comme le Geometral..., Paris, De l'Imprimerie de Pierre Des-Hayes, 1648.

32. René Descartes, « Discours quatrième », dans La dioptrique [1637], Paris, Gallimard, 2009.

33. Je commente ces auteurs dans L'époque des appareils, 2004. En part. : Arendt et la politique.

34. Hans Belting, Florence et Bagdad. Une histoire du regard entre orient et occident, op. cit.

\section{RÉSUMÉS}

Sophie-Aurore Roussel a présenté ici même le programme de sa thèse qui vise à confronter le théâtre grec pré-aristotélicien avec sa reprise sur la scène contemporaine. Elle fait appel, pour mieux caractériser le théâtre grec, aux ouvrages de Florence Dupont. Cette dernière, à la différence des Romantiques et des théoriciens de la catharsis, institue ce-dernier comme un véritable appareil, dont les textes qui subsistent (Euripide, Sophocle, Eschyle, etc.) sont les produits esthétiques, objets de multiples mises en scène contemporaines. Avant de fétichiser ces textes, les "tragédies", il convient donc de décrire ce proto-appareil d'un point de vue architectural et technique, car les machines au cœur de sa mise en œuvre sont essentielles. Elles permettront de générer des figures analogiques. Si l'on peut parler, à son sujet, de proto-appareil pour l'Occident, c'est que le théâtre grec appareille un ordre de discours fondamental, une cosmétique, qui est toujours la nôtre pour l'essentiel. On peut le considérer comme un appareil originaire au sens où il constituera l'infrastructure des appareils secondaires qui se développeront à partir du maniement de la perspective, à la Renaissance italienne. Ces appareils projectifs, comme le musée, la photographie, le cinéma, etc. auront toujours, d'une manière ou d'une autre, à prendre en considération le théâtre, qui est leur socle. La projection, même quantitative, géométrique, doit donc être considérée comme un mode de l'analogie définit par 
Kant. Mais comme le montre la Dioptrique de Descartes, il ne s'ensuit pas qu'elle doive être imitative. Ce qui représente peut être abstrait, comme le sont les entailles de la gravure. Dès lors, il n'y a pas de véritable coupure entre la peinture classique et la peinture abstraite, ni entre l'écriture projective et l'écriture numérique.

INDEX

Mots-clés : théâtre grec, appareil, analogie, projection, Florence Dupont, Florens Christian Rang, Walter Benjamin, Jorge Louis Borges, Descartes, Sigmund Freud, Jean-François Lyotard, Hans Belting

\section{AUTEUR}

JEAN-LOUIS DÉOTTE †

Professeur émérite de philosophie, université Paris 8 Saint-Denis, MSH Paris Nord 